\title{
MEMBERSHIP LIST
}

(C), denotes Charter Member; (F), Fellows; (H), Honorary Member. The figures in parenthesis indicate the year of election to membership. Organization names are listed when the business address is given.

Аввот, E. Stanley, M.D., 29 Gloucester St., Boston, Mass. (-) ('29).

Ackerly, Spafford, M.D., Mental Hygiene Clinic, 610 S. Floyd St., Louisville, Ky. (B) ('31).

Ackerson, Luton, Ph.D., Institute for Juvenile Research, 907 S. Lincoln St., Chicago, Ill. (E) ('29'). Adams, Walter A., M.D., ${ }_{4} 6_{41}$ S. Wabash Ave., Chicago, Ill. ('35).

Alexander, Franz, M.D., Institute for Psychoanalysis, 43 E. Ohio St., Chicago, Ill. (E) ('31).

Allen, Mrs. Cornelia D. H., School of Social Work, University of Buffalo, Buffalo, N. Y. (2) ('30).

Allen, Elizabeth, Department of Public Welfare, I 40 W. Willow St., Syracuse, N. Y. ('29).

Allen, Frederick H., M.D., Child Guidance Clinic, I7I I Fitzwater St., Philadelphia, Pa. (E) ('26).

Alpern, Evelyn M.D., The Institute of the Pennsylvania Hospital, in I N. 49th St., Philadelphia, Pa. ('33).

Amsden, George S., M.D., i 5 E. 67th St., New York, N. Y. (E) ('32).

Anderson, Forrest N., M.D., Child Guidance Clinic, I325 W. Adams Blvd., Los Angeles, Cal. (P) ('29).

Anderson, V. V., M.D., The Spruces, Staatsburg-on-Hudson, N. Y. (B) (C).

Arrington, Winifred W., National Committee for Mental Hygiene, 50 W. 50 th St., New York, N. Y. (F) ('30).

Arthur, Grace, Ph.D., Amherst H. Wilder Child Guidance Clinic, 279 Rice St., St. Paul, Minn. (F) ('32).

August, Harry E., M.D., ro56 Maccabee Bldg., Detroit, Mich. ('33).

Baker, Amos T., M.D., P.O. Box G, Briarcliff Manor, N. Y. (') ('25).

Bancrofr, Harold A., M.D., I 79 Allyn St., Hartford, Conn. ('26).

Barbee, Margaret, Henry Watson Children's Aid Society, 31 S. Calvert St., Baltimore, Md. ('33).

Barrows, Franklin W., M.D., 26 Wood Pl., Dumont, N. J. ('32).

Bartelme, Phyllis, Ph.D., ioi 5 Garden St., Santa Barbara, Calif. (E) ('29).

Bartemeier, Leo H., M.D., 8-259 General Motors Bldg., Detroit, Mich. (i) ('29).

BassetT, Clara, National Committee for Mental Hygiene, 5o W. 50 th St., New York, N. Y. (1) ('28).

Beck, Samuel J., Ph.D., Boston Psychopathic Hospital, 74 Fenwood Rd., Boston, Mass. (7) ('29).

Blanchard, Myron B., Edenwald School for Boys, 225th St. and Boston Rd., Bronx, N. Y. ('34).

Blanchard, Phyllis, Ph.D., Child Guidance Clinic, I7I I Fitzwater St., Philadelphia, Pa. (1) ('30).

Bradley, Charles, M.D., Emma Pendleton Bradley Home, E. Providence, R. I. ('36).

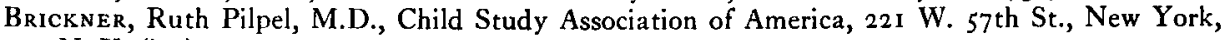
N. Y. ('34).

Bridgman, Olga, M.D., I 380 Monterey Blvd., San Francisco, Cal. (E) ('25).

Bridgman, Ralph P., National Council of Parent Education, 60 E. 42nd St., New York, N. Y. (E) ('31).

Brinker, Dorothy, The Jefferson, Apt. 309, Jefferson and Third St., Niagara Falls, N. Y. ('32).

Broadwin, Isra T., M.D., i 6 W. 59 th St., New York, N. Y. (E) ('30).

Brockbank, Thomas W., M.D., Catholic Charities Guidance Institute, I 81 E. 68th St., New York, N. Y. ('33).

Bronner, Augusta F., Ph.D., Judge Baker Guidance Center, $38 \frac{1}{2}$ Beacon St., Boston, Mass. (F) ('25).

Brown, Frederick W., 69 Cambridge Ave., Garden City, N. Y. ('28).

Brown, Muriel W., Ph.D., National Council of Parent Education, 60 E. 42nd St., New York, N. Y. ('3o).

Brown, Sanger, II, M.D., Cold Spring-on-Hudson, N. Y. (F) ('28).

Burling, Temple, M.D., 785 Locust St., Winnetka, Ill. (F) ('33).

Challman, S. A., M.D., Child Study Department, Board of Education, Central Ave., and S.E. Fourth St., Minneapolis, Minn. ('35).

Chamberlain, Herbert E., M.D., Bobs Roberts Memorial Hospital, 920 E. 59th St., Chicago, Ill. (D) ('27).

Chapman, Ross McC., M.D., Sheppard and Enoch Pratt Hospital, Towson, Md. ('28). 
Childers, Asher T., M.D., The Central Clinic, Cincinnati General Hospital, Cincinnati, O. (B) ('31).

Cноч, Li-chiu, Social Service Department, Peiping Union Medical College Hospital, Peiping, China ('36).

Clark, Mary Augusta, National Committee for Mental Hygiene, 50 W. 5oth St., New York, N. Y. (F) ('30).

Clarke, Eric Kent, M.B.(Tor.), Strong Memorial Hospital, Rochester, N. Y. (F) ('27).

Clifton, Eleanor, 26 W. 9th St., New York, N. Y. ('27).

Cochran, Mrs. Mary Lue, Allentown State Hospital, Allentown, Pa. ('3i).

Cogrint, Harvie DeJ., M.D., Children's Memorial Clinic, 100 I E. Clay St., Richmond, Va. (E) ('28).

Conson, Harold F., M.D., Austen Riggs Foundation, Stockbridge, Mass. ('31).

Couch, Mildred Warden, M.D., Cromwell Hall, Cromwell, Conn. ('31).

CRowell, Sarah Eyre, Edgemont, Delaware Co., Pa. ('36).

Cummings, Charles K., Jr., 153 West St., Kendal Green, Mass. ('32).

Cunningham, James M., M.D., Bureau of Mental Hygiene, Department of Health, Hartford, Conn. ('33).

Dawley, Almena, Child Guidance Clinic, I 7 I I Fitzwater St., Philadelphia, Pa. (1) ('3I).

DeBerry, Ellett M., M.D., University of Minnesota, Minneapolis, Minn. (B) ('29).

Dolt, Edgar A., Ph.D., The Training School, Vineland, N. J. (F) ('25).

Dombey, Edith H., Juvenile Court, East 22nd St. and Cedar Ave., Cleveland, O. ('34).

Doody, William M., M.D., I9 Bentley Ave., Jersey City, N. J. ('35).

Dummer, Mrs. W. F., 679 N. Michigan Ave., Chicago, Ill. (H) ('26).

Ebaugh, Franklin G., M.D., 1788 Glencoe St., Denver, Colo. (3) ('29).

Erster, Edwin R., M.D., 43 E. Ohio St., Chicago, Ill. () ('29).

ElKind, Henry B., M.D., Massachusetts Society for Mental Hygiene, 3 Joy St., Boston, Mass. (F) ('28).

Emery, E. Van Norman, M.D., Yale University, 333 Cedar St., New Haven, Conn. (E) ('3I).

Englander, Charles, M.D., 4I Hillside Ave., Newark, N. J. ('3I).

Ewerhardt, Paul J., M.D., Child Guidance Clinic, I 464 Columbia Rd., N.W., Washington, D. C. (E) ('30).

Ferguson, William G., M.D., Child Guidance Clinic, Pine Ave. and inth St., Niagara Falls, N. Y. ('30).

Foertmeyer, A. W., M.D., 505 Walnut St., Cincinnati, O. ('27).

Foster, Sybil, Child Welfare League of America, i $3 \circ$ E. 22nd St., New York, N. Y. ('30).

Frank, Lawrence K., General Education Board, 49 W. 49 th St., New York, N. Y. (i) (' 32 ).

FRENCH, Mrs. Lois Meredith, 34 Kearney St., Newark, N. J. ('33).

Fries, Margaret E., M.D., 9 West 82nd St., New York, N. Y. ('36).

FRY, Clem C., M.D., Iog College St., Yale Sta., New Haven, Conn. ('31).

Fuller, Earl W., M.D., 8 Mill Rd., Morristown, N. J. (R.F.D.) ('3I).

Garretr, Annette, 8 College Hall, Northampton, Mass. (' 31 I).

Gerard, Margaret W., M.D., Division of Psychiatry, University of Chicago, Chicago, Ill. ('3I).

Geseld, Arnold, M.D., 185 Edwards St., New Haven, Conn. (F) ('31).

Gitelson, Maxwell, M.D., Institute for Juvenile Research, 907 S. Lincoln St., Chicago, Ill. ('36).

Goldman, Julia, Hebrew Sheltering Guardian Society, Pleasantville, N. Y. ('33).

Gould, Prof. Miriam C., Vassar College, Poughkeepsie, N. Y. ('33).

GRIfFIN, D. P., M.D., I 278 E. Main St., Bridgeport, Conn. ('26).

Grossman, Grace, Jewish Board of Guardians, 228 E. 19th St., New York, N. Y. ('3I).

Grossman, Royal G., M.D., 2245 Arthur Ave., Lakewood, O. ('28).

Hagan, Margaret Wood, American Red Cross, St. Elizabeths Hospital, Washington, D. C. ('36).

Hagman, Elmer R., Ph.D., Hartley_Salmon Clinic, 488 Main St., Hartford, Conn. ('33).

Hardcastle, Douglas Noel, M.D., 2, Lyndhurst Road, N.W. 3, London, England ('34).

Harrington, Milton, M.D., Napanoch, N. Y. ('32).

Harrison, Frances, 1520 Spruce St., Philadelphia, Pa. ('32). 
Hartwell, Samuel W., M.D., Buffalo University Medical School, 24 High St., Buffalo, N. Y. (F) ('29).

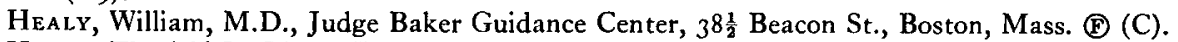

Hegge, Thorleif G., Ph.D., Wayne County Training School, Northville, Mich. ('35).

Heldt, Thomas J., M.D., Henry Ford Hospital, Detroit, Mich. ('29).

Helgesson, Uno H., M.D., Smith College, Northampton, Mass. ('3I).

Hennessy, Maurice A. R., M.D., Juvenile Court, Juvenile Court Bldg., Cleveland, O. ('3I).

Hill, Julia Ford, M.D., Worcester Child Guidance Clinic, 2I Catherine St., Worcester, Mass. ('35). Hrncks, Clarence M., M.D., Canadian National Committee for Mental Hygiene, i I I St. George St., Toronto, Canada ('33).

Hincks, Elizabeth M., Ph.D., 58 Washington Ave., Cambridge, Mass. ('32).

Holmer, Paul, M.D., I6 I9 Olive Ave., Reading, Pa. ('34).

Horton, Charles B., M.D., Mental Hygiene Center, 225 N. Jefferson St., Dayton, O. ('33).

Igel, Amelia M., 2 Io E. 68th St., New York, N. Y. ('35).

JAckson, James D., 27 N. Dean Ave., Trenton, N. J. ('30).

JAEger, Martha H., College of William and Mary, gor W. Franklin St., Richmond, Va. ('33).

Jaenike, R. C. A., M.D., Child Guidance Clinic, Strong Memorial Hospital, Rochester, N. Y. ('33).

Janvier, Carmelite, 3307 Prytania St., New Orleans, La. ('35).

Jefrerson, Roland A., M.D., 208 E. Wisconsin Ave., Milwaukee, Wis. ('33).

Johnson, Eleanor Hope, Ph.D., The Hartford School of Religious Education, Hartford, Conn. (E) ('26).

Johnson, George S., M.D., 2238 Hyde St., San Francisco, Calif. ('3o).

Joslyn, June J., New Jersey State Hospital, Trenton, N. J. ('34).

Kasanin, Jacob, M.D., Michael Reese Hospital, 29th and Ellis Ave., Chicago, Ill. (F) ('26).

Kawin, Ethel, 5600 Blackstone Ave., Chicago, Ill. (F) ('32).

Kenworthy, Marion E., M.D., I035 Fifth Ave., New York, N. Y. (E) ('24).

Kimball, Everett, Ph.D., I 86 Elm St., Northampton, Mass. (B) ('3o).

KirchweY, Prof. George W., io5 E. 22nd St., New York, N. Y. (H) ('26).

Kirkpatrick, M. E., M.D., Worcester Child Guidance Clinic, 2i Catherine St., Worcester, Mass. (E) ('31).

Kovarsky, Marcel, Jewish Board of Guardians, 228 E. 19th St., New York, N. Y. ('3I).

LaBine, Alfred C., M.D., 8-259 General Motors Building, Detroit, Mich. ('28).

LaMAR, Norvelle C., M.D., 210 E. 68th St., New York, N. Y. (E) ('30).

Langrord, William Siddon, M.D., Babies Hospital, 167th St. and Broadway, New York, N. Y. ('36).

Langner, Helen P., M.D., Vassar College, Poughkeepsie, N. Y. ('29).

Larson, John A., M.D., 907 S. Lincoln St., Chicago, Ill. ('3I).

Laughead, Mary B., 30 W. 54 th St., New York, N. Y. ('30).

Lawton, George, Ph.D., 35 W. 82nd St., New York, N. Y. ('35).

LEe, Porter R., New York School of Social Work, 122 E. 22nd St., New York, N. Y. (E) ('29).

Leonard, Christine M., M.D., 30 W. 54th St., New York, N. Y. (F) ('24).

Leonard, Shirley, Bureau of Child Guidance, P.S. \#59,228 E. 57 th St., New York, N. Y. (1) ('31).

Levin, Max, M.D., Harrisburg State Hospital, Harrisburg, Pa. ('30).

LevY, David M., M.D., 136 E. 57 th St., New York, N. Y. (1) (C).

LevY, John, M.D., 7I E. 77th St., New York, N. Y. (ㅇ ('29).

Lewin, Bertram D., M.D.; 25 Fifth Ave., New York, N. Y. ('31).

Li в веy, Betsey, 3I I S. Juniper St., Philadelphia, Pa. ('3I).

Lippman, Hyman S., M.D., Amherst H. Wilder Child Guidance Clinic, 279 Rice St., St. Paul, Minn (F) ('28).

Liss, Edward, M.D., i 30 E. 39th St., New York, N. Y. (E) ('32).

Lithauer, Donah B., 520 W. I Ioth St., New York, N. Y. ('32).

LitTle, Harry M., M.D., Child Guidance Center, 3604 Victoria St., Pittsburgh, Pa. ('35).

Lloyd, Wilma, F., i 7 E. 96th St., New York, N. Y. ('31).

Lonergan, Michael P., M.D., Manhattan State Hospital, Ward's Island, New York, N. Y. ('3o) 
Lorand, Alexander S., M.D., i 5 E. 86th St., New York, N. Y. ('33).

LotT, George M., M.D., Howard, R. I. ('33).

Lowrey, Lawson G., M.D., 28 W. 54th St., New York, N. Y. (E) (C).

Luckey, Bertha M., Ph.D., Board of Education, Cleveland, O. ('31).

Luérrs, Leslie E., M.D., 4o W. 9th St., New York, N. Y. ('29).

Lurie, Louis A., M.D., Doctors Building, Cincinnati, O. († ('26).

Lyman, Richard, M.D., Peiping Union Medical College, Peiping, China ('34).

MacDonald, Martha Wilson, M.D., Michael Reese Hospital, 29th and Ellis Avenue, Chicago, Ill. ('33).

McCartney, James L., M.D., 380 Main St., Catskill, N. Y. ('30).

McClure, Jeannette E., Children's Center, 3743 Brush St., Detroit, Mich. ('32).

McClure, Mrs. Robert, Child Guidance Center, 3604 Victoria St., Pittsburgh, Pa. ('31).

McCord, Clinton P., M.D., 20 Willett St., Albany, N. Y. (F) ('26).

Maeder, LeRoy, M.A., M.D., Chancellor Hall, 204 S. 13 th St., Philadelphia, Pa. ('29).

Malcove, Lillian, M.D., 245 E. 72 nd St., New York, N. Y. ('31).

Markey, Oscar B., M.D., 7oI6 Euclid Ave., Cleveland, O. (†) ('28).

Marshall, Berry C., M.D., 419 Hawthorn Rd., Roland Park, Baltimore, Md. ('33).

Marshall, Helen, 61o Livermore St., Yellow Springs, O. ('34).

Martin, A. Leila, Ph.D., Child Study Department, Board of Education, 13 Fitzhugh St., S., Rochester, N. Y. ('26).

Mason, Sue H., 1358 Lincoln Ave., St. Paul, Minn. ('35).

Mayer, Edward E., M.D., Medical Bldg., Pittsburgh, Pa. ('34).

Mertzer, H., Ph.D., Psychological Service Center, 5245 Waterman Ave., St. Louis, Mo. (-) ('31).

Menninger, Karl A., M.D., Menninger Clinic, 36i 7 W. 6th Ave., Topeka, Kans. (1) (C).

Menninger, William C., M.D., Menninger Clinic, 36i 7 W. 6th Ave., Topeka, Kans. ('35).

Meyer, Adolf, M.D., Johns Hopkins Hospital, Baltimore, Md. (P) ('24).

Mitchell, William T. B., M.D., McGill University, Montreal, Canada (E) ('26).

Mонr, Mrs. Esther J., 43 E. Ohio St., Chicago, Ill. ('32).

Мон R, George J., M.D., 43 E. Ohio St., Chicago, Ill. (E) ('26).

Montague, Helen, M.D., 27 W. 9 th St., New York, N. Y. (2) ('24).

Moore, Madeline U., 45 Christopher St., New York, N. Y. ('33).

Morsh, Mrs. Edith Symmes, University Lodge, University Hill, Vancouver, B. C., Canada ('28).

Moulton, Bryant E., M.D., Judge Baker Guidance Center, $38 \frac{1}{2}$ Beacon St., Boston, Mass. ('31).

Moulton, Phyllis, 3I 5 E. 68th St., New York, N. Y. ('31).

Moyle, Henry B., M.B. (Tor.), Hartley_Salmon Clinic, 488 Main St., Hartford, Conn. (F) ('29).

Murphey, Bradford J., M.D., Child Guidance Clinic, Bemis-Taylor Foundation, $\mathrm{O}_{4}$ E. Rio Grande St., Colorado Springs, Colo. (i) ('29).

Murray, Willa L., Apt. 5I3, Cavalier Hotel, Washington, D. C. ('3o).

Muskat, Caroline J., c/o Dr. Muskat, Marietta, O. ('32).

Nelson, William, M.D., 5449 Delmar Blvd., St. Louis, Mo. ('29).

Neumann, Frederika, ig W. Ioth St., New York, N. Y. ('31).

Newell, Horatio W., M.D., 7106 Wardman Rd., Govans P.O., Baltimore, Md. (F) ('3o).

Oberndorf, Clarence P., M.D., i I 2 W. 59th St., New York, N. Y. (E) ('31).

O'Brien, Frank J., M.D., Bureau of Child Guidance, P.S. \#59, 228 E. 57th St., New York, N. Y. (F) ('25).

Otson, Elma, Family Welfare Association, 1802 Maple Ave., Evanston, Ill. (1) ('29).

Osson, Willard C., Ph.D., School of Education, University of Michigan, Ann Arbor, Mich. (A) ('29).

Orgel, Samuel Z., M.D., 667 Madison Ave., New York, N. Y. (F) ('29).

Parker, Z. Rita, M.D., 115 E. 6ist St., New York, N. Y. ('28).

Patry, Frederick L., M.D., State Department of Education, Albany, N. Y. (E) ('32).

Paynter, Richard H., Ph.D., 395 Grand Ave., Brooklyn, N. Y. (i) ('30).

Pearson, Gerald H. J., M.D., Pennsylvania Hospital i i N 49th St., Philadelphia, Pa. (E) ('30).

PePpard, S. Harcourt, M.D., Bureau of Child Guidance, P.S. \#103, I66th St. and 65th Ave., Flushing, N. Y. ('3I). 
Perkins, Nellie, Ph.D., 3745 Clairmount Ave., Detroit, Mich. ('25).

Perry, Doris E., 515 W. 122nd St., New York, N. Y. ('30).

Pratt, George K., M.D., I 22 E. 76 th St., New York, N. Y. (E) ('27).

Preston, George H., M.D., 33o N. Charles St., Baltimore, Md. (1) ('29).

Prirchert, Henry L., Ph.D., Southern Methodist University, Dallas, Tex. ('3I).

Putnam, Marian C., M.D., Institute of Human Relations, Yale University, 333 Cedar St., New Haven, Conn. ('32).

Quinlan, Gertrude, Catholic Charities Guidance Institute, 18I E. 68th St., New York, N. Y. ('35).

Rademacher, E. S., M.D., 442 Temple St., New Haven, Conn. (E) ('27).

RaphaEl, Theophile, M.D., University Health Service, University of Michigan, Ann Arbor, Mich. (F) ('26).

Reeve, George H., M.D., 8314 Euclid Ave., Cleveland, O. (F ('26).

Reynolds, Bertha C., 3947-48th St., Long Island City, N. Y. (E) ('30).

Rich, Gilbert J., M.D., Mental Hygiene Clinic, 2430 W. Wisconsin Ave., Milwaukee, Wis. (P) ('29).

Richa Rds, Esther Loring, M.D., Johns Hopkins Hospital, Baltimore, Md. (F) ('29).

Richmond, Frank C., M.D., State Board of Control, Madison, Wis. ('35).

Richmond, Winifred, Ph.D., St. Elizabeths Hospital, Washington, D. C. ('36).

Roв B, Christine C., American Association of Social Workers, 130 E. 22nd St., New York, N. Y. (B) ('28).

Robie, Theodore R., M.D., 3o Norman Rd., Upper Montclair, N. J. ('30).

Rockwel., Alice J., Ph.D., 6oI W. Lombard St., Baltimore, Md. ('33).

Rogers, Carl R., Ph.D., Department of Child Study, Rochester Society for the Prevention of

Cruelty to Children, 158 Plymouth Ave., N., Rochester, N. Y. ('34).

Roor, June A., Family Welfare Bureau, 3 I I State St., Schenectady, N. Y. ('3I).

Root, Manly B., M.D., The National Training School for Boys, Bladensburg Rd., Washington, D. C. (1) ('29).

Rosenblum, Deborah F., Jewish Board of Guardians, 228 E. Igth St., New York, N. Y. ('31).

Rosenzweig, Saul, Ph.D., Worcester State Hospital, Worcester, Mass. ('35).

Roth, William F., Jr., M.D., Williamson County Child Guidance Study, Franklin, Tenn. ('35).

Ruggles, Arthur H., M.D., Butler Hospital, Providence, R. I. ('29).

Scha piro, Joseph, M.D., Bureau of Child Guidance, 712 Palisade Ave., Union City, N. J. ('35).

Schroeder, Paul L., M.D., Institute for Juvenile Research, 907 S. Lincoln St., Chicago, Ill. (1) ('30).

Schumacher, Henry C., M.D., Child Guidance Clinic, 2525 Euclid Ave., Cleveland, O. (P) ('26).

Schwartz, Louis A., M.D., 423 Professional Bldg., Io Peterboro St., Detroit, Mich. ('31).

Schwarzenberg, Edith A., New York School of Social Work, 122 E. 22nd St., New York, N. Y. ('3I).

Scoville, Mildred C., The Commonwealth Fund, 4I E. 57th St., New York, N. Y. (P) ('26).

Segerer, Elizabeth, Ph.D., I 47 Mason St., Cincinnati, O. ('28).

Selling, Lowell S., M.D., 8545 Second Blvd., Detroit, Mich. ('30).

Senior, Rose D., M.D., 56r 2 Kenwood Ave., Chicago, Ill. ('35).

Shakow, David, Box 57, Worcester, Mass. (' 30 ).

Shimberg, Myra E., Ph.D., Judge Baker Guidance Center, $38 \frac{1}{2}$ Beacon St., Boston, Mass. ('32).

Stewers, Albert B., M.D., Medical Arts Bldg., Syracuse, N. Y. (E) ('29).

Silliman, Marguerite, Box 411 , Coral Gables, Fla. ('36).

Silverman, Baruch, M.D., 53I Pine Ave., W., Montreal, Canada ('31).

Skinner, Anna M., M.D., 4 Bates Rd., Watertown, Mass. ('29).

Slawson, John, Ph.D., Jewish Board of Guardians, 228 E. Igth St., New York, N. Y. ('36).

Smalley, Ruth E., 13 Fitzhugh St., S. Rochester, N. Y. ('36).

SмITH, Barry C., The Commonwealth Fund, 4I E. 57th St., New York, N. Y. (H) ('30).

Sмiтн, Groves Blake, M.D., Beverly Farms, Inc., Godfrey, Ill. (1) ('30).

SPEnCER, W. Douglas, 1240 W. Broadway, Hewlett, L. I., N. Y. ('32).

Stein, Florence C., M.D., Ward's Island, New York, N. Y. ('30).

Stephenson, Charles W., M.D., 179 Allyn St., Hartford, Conn. ('32).

Stevenson, George S., M.D., National Committee for Mental Hygiene, 50 W. 50 th St., New York, N. Y. (B) (C). 
Stoy, Edward G., Ph.D., 68 Post St., San Francisco, Calif. ('31).

Stranahan, G. Marion, M.D., 6i E. 86th St., New York, N. Y. ('3I).

Strecker, Edward A., M.D., Pennsylvania Hospital, i i I N. 49th St., Philadelphia, Pa. (E) ('24).

Sullivan, Harry Stack, M.D., 158 E. 64th St., New York, N. Y. (F) ('30).

Sumne R, Mary C., 330 E. 43rd St., New York, N. Y. ('26).

Svendsen, Margaret T., Institute for Juvenile Research, 907 S. Lincoln St., Chicago, Ill. ("34).

Swifr, Sarah H., 1035 Fifth Ave., New York, N. Y. (F) ('26).

TAFr, Jessie, Ph.D., 31 I S. Juniper St., Philadelphia, Pa. (A) ('25).

TAussig, Helen P., I 4 E. 75 th St., New York, N. Y. ('3I).

Thompson, Lloyd J., M.D., Duke University School of Medicine, Durham, N. C. (F) ('29).

Thurrott, John C., M.D., 29 Washington Square West, New York, N. Y. ('31).

Tiesout, Harry M., M.D., 215 Milbank Ave., Greenwich, Conn. (E) ('28).

Tracy, Anna Belle, School of Applied Social Sciences, Western Reserve University, 2117 Adelbert Rd., Cleveland, O. (E) ('3o).

Truitr, Ralph P., M.D., Mental Hygiene Society of Maryland, 6oI W. Lombard St., Baltimore, Md. (1) ('30).

Tulchin, Simon H., i36 E. 57th St., New York, N. Y. () ('28).

Ulrich, Carl F., M.D., I668 E. I I 7 th St., Station E., Cleveland, O. ('30).

Vedder, Mrs. Sarah V. D., A.I.C.P., Iog E. 22nd St., New York, N. Y. ('31).

Wagner, Carl P., M.D., Hartford Retreat, 200 Retreat Ave., Hartford, Conn. ('34).

Washburn, Ruth W., I 45 Deepwood Drive, New Haven, Conn. ('32).

Warson, Goodwin, Ph.D., Teachers College, 525 W. 120 th St., New York, N. Y. ('33).

Watson, Maud E., Ph.D., 2240 W. Grand Blvd., Detroit, Mich. ('34).

Webber, Alice J., 310 W. 97 th St., New York, N. Y. ('3I).

Weill, Blanche C., Ed.D., 470 W. 24th St., New York, N. Y. ('35).

Whitehead, Mary L., 235 E. 22nd St., New York, N. Y. ('3o).

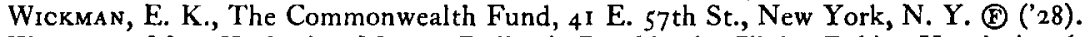

Wrckman, Mrs. Katharine Moore, Pediatric-Psychiatric Clinic, Babies Hospital, I67th St. and Broadway, New York, N. Y. ('29).

Wiedman, Otto G., M.D., I79 Allyn St., Hartford, Conn. (E) ('26).

Wile, Ira S., M.D., 264 W. 73rd St., New York, N. Y. (1) ('26).

Williams, Frankwood E., M.D., 44 W. I2th St., New York, N. Y. (1) ('24).

Witmer, Helen Leland, Ph.D., 317 E. I 7 th St., New York, N. Y. (B) ('34).

Witmer, Prof. Lightner, University of Pennsylvania, Philadelphia, Pa. ('25).

Woolley, Lawrence F., M.D., 6 Dixie Drive, Towson, Md. ('32).

Yepsen, Lloyd N., Ph.D., New York University, Washington Square East, New York, N. Y. ('28).

Zachry, Caroline B., Ph.D., i7 E. 96th St., New York, N. Y. (E) ('30).

Zilвoorg, Gregory, M.D., 14 E. 75th St., New York, N. Y. ('32).

Zorbaugh, Harvey, School of Education, New York University, Washington Square East, New York, N. Y. (1) ('29). 